\title{
Discussion on the Construction of Ecosystem of Innovation and Entrepreneurship Education in the Colleges and Universities
}

\author{
Chunfeng Jiang ${ }^{1, a^{*}}$ and Yulan Zhao ${ }^{2, b}$ \\ ${ }^{1,2}$ Jilin Agricultural Science and Technology University, Jinlin China \\ ahero_jcf@126.com, bllbird@126.com
} Keywords: Ecological system; Cooperative operation; Education innovation; Operation
mechanism

\begin{abstract}
This paper discusses the operating mechanism of the ecological system of the innovative talents in universities and colleges with the interaction among the elements of the ecological system. In this paper, we believe that the universities, the objects of education, the education circumstances and the carriers respectively play the role of producers, consumers, decomposers and the inorganic environment. By comparing the ecological operation of the education system of the innovation talents between Chinese and American higher education, this article puts forward to promote the full development of the independent function of various factors and the positive cycle interaction among the elements, to promote the development of each element in the educational system more actively, and to enhance the motive power of communicating the internal and the external.
\end{abstract}

\section{Introduction}

It is the important strategic decision to construct an innovative country, which is made by the national leaders according to the trend of the world's technological development and China's unique national conditions. This kind of decision can make our country be able to face and meet the challenge of knowledge economy better, and it is the important prerequisite to transform facing the future development. To accelerate the implementation of this important decision, the key lies in the emergence of innovative talents and the improvement of the quality of entrepreneurship and innovation education. As a new type of worker, the invisible abilities provided to individuals, such as thinking and ideology, is the practical significance to stimulate and reconstruct the entrepreneurship and innovation education. Entrepreneurship and innovation education is a very complex task to stimulate and reconstruct people's potential, and it cannot be completed by the educators' personal abilities. Higher institutions should effectively organize and collect all kinds of factors and supportive systems that can serve for the cultivation of innovative talents, and make these elements interact positively under certain conditions to construct the soil environment for cultivating innovative talents, which is the educational ecosystem of the innovative talents in colleges and universities.

\section{The Significance of Constructing the Educational Ecosystem of the Entrepreneurial Talents}

According to the explanation in the "Ci Hai" 1999 edition, "ecological system" also known as "ecosystem", refers to the interactions between the natural system and the geographical environment of the biological communities. It consists of four basic components, namely, the inorganic environment, biological producer, consumer, decomposition, and the links between the biological food chain. The energy flow and biological material circulation of the core ecosystem is the crucial function and characteristics of the ecosystem, and its structure constraints the natural ecosystems, the function of biomass production, and the influence of self-regulation and control towards the environment. In July 2004, the United States Competitiveness Commission explores the social factors of innovation model from the aspect of the States in the interim report, which entitled "Innovative America: To Challenge and Maintain the Prosperity in a constantly Changing World". There is a lot of space for the understanding of innovation ecosystem for the current position in the 
academic. There are hundred schools of thought lies in the discussion of the connotation and extension, and there is no unified conclusion for the innovation ecosystem model which needs different levels and different disciplines. However, the balance of the elements in the "business ecosystem" which is generally recognized by the scholars should be in accordance with the main body of innovation, natural integration of the diversification, and the development of the basic content of the system. This paper describes how the Massachusetts Institute of Technology (MIT) forms a complete ecosystem of entrepreneurial incubators, and uses a large number of startups and talents in the system.

Inspired by this concept, many domestic scholars use the "ecosystem" to study the operational advantages of the American University's entrepreneurial ecosystem, and to compare with the domestic universities and colleges. The conclusions drawn by the scholars are roughly the same, he said, "entrepreneurial ecosystem" is mainly composed of a market like ecosystem of several important factors, such as, institutional policies of the state, financing, human capital, the school based extracurricular activities based on school and local culture, etc. The interactions of each element and the structure of cooperation is the source to encourage and support entrepreneurial activity, and ecosystem functioning well is the guarantee of high density and high efficiency of production. The research described here is "ecosystem of innovation and entrepreneurship education in the colleges and universities", as well as two different ecological systems. The focus of this research is to foster innovation and entrepreneurship education ecosystem based on the core of the university. In other words, on the one hand, it is to explore what kind of educational policy is to develop the university, in which the implementation of education tools support creating education conditions, so that education can establish the cultural atmosphere of innovation and entrepreneurship ecosystem; in the other hand, it should lead the ecological exposure system training objects, the students' feedback interactive environment (in ecosystem power) and the time environment and government support (ecosystem dynamics) combined to promote the sustainable development of a harmonious ecological system.

For this problem, in recent years, some domestic scholars have carried out a study. Liu Zhenya made the comparative study of Chinese and American entrepreneurs to foster the ecological education introduce the advantageous and the advanced American entrepreneurship education ecological culture, which achieved the aim that ecological culture of entrepreneurship education can learn from experience and enlightenment. In the view of the attitude of Feng Yong and students in Jiangsu Province Chiron towards entrepreneurial culture, entrepreneurial intention and the problems of colleges and universities carrying out entrepreneurship education and entrepreneurial motivation, three types of 15 universities and colleges in Jiangsu Province make an investigation. And it put forwards to construct strategic ecosystem according to the key findings of entrepreneurship education in the colleges and universities. Xu Jifang proposes to construct ecosystem of entrepreneurship education, whose all the relevant link parts form one step of an interactive system. And then one after another, it forms the important content from various aspects, and improving the education system is an important part to build the ecological system. According to the results of the survey, the construction of innovation and entrepreneurship is a full and comprehensive view in the perspective of entrepreneurial education. But in the positioning of entrepreneurship education, lacking of concise and clear main factors responsible for the formation of innovation and entrepreneurship education itself will be discussed. In order to solve the above problems, this paper will focus on the study of the ecological construction of "annual entrepreneurship education in colleges and universities" between China and America, discuss the interaction between the factors, and provide a theoretical guidance for the construction of system in domestic colleges and universities.

There are following three types of this kind of research: first, it is in the cultural form of innovation, such as definite mechanism of driving system, deeper understanding of the relationship among the various elements between the internal and external power, and facilitating the positioning of various elements and expand the direction; second, it is in education, such as to promote innovation and entrepreneur spirit, comparing the structure and content of the United States and 
European countries purposely from different directions and layers, and learning from their experience; third, it is conducive to establish the concept of innovative record among professionals in global educative mechanism. And it is conducive to the policy formulation of educational mode in university and the sustainable development of the system itself by focusing on integrating the effectiveness of interactions among all elements.

\section{The Structure, Elements and Driving Force of Entrepreneurship and Innovation Talents in Colleges and Universities}

The main characteristic of "ecosystem" is that life activities of the body to perform their respective roles and symbiotic exchange in inorganic environment to promote the comprehensive, sustained and healthy development of energy. The reason is that the metaphor of the "ecosystem" is to establish the model of innovation and entrepreneurship education environment. Because the former is a dynamic balance and the ideal model of sustainable systems, and to cultivate of education and innovation talents precisely needs to establish such a system. Based on the relationships between the various elements of the ecological system and the functional model of the ecosystem in the education, it metaphors the elements of the innovation and entrepreneurship education.

Producers. The producers are the core elements of the ecological system, and they produce materials and energy for themselves and other organisms through photosynthesis and chemical synthesis. That's to say, in a closed ecosystem, the production rate of producers is greater than the consumers or decomposition agents. This definition requires that the core elements we choose in the ecosystem of entrepreneurship and innovation education also have the ability to support themselves and provide the source of other elements. Only the university can take on the dual mission in the core of the language, so the university is the producer of the ecosystem of entrepreneurship and innovation education.

Consumers. As a part of the food chain in the ecological system, consumers themselves can not engage in production activities, and they can only achieve self-survival by consuming other organisms. Consumers can be divided into different levels relied on their own position, as the middle part of the conversion of material and energy in the ecosystem. Consumers rely on the two key concepts of "consumer" and "producer", and decide the definition of the "rely" relationships among the objects of the eco system of innovation and entrepreneurship education combining with the producers. According to the Ministry of Education vigorously promote the self-employment career of innovation and entrepreneurship education in colleges and universities (Education Office [2010] 3 ), the innovation and entrepreneurship education should be oriented to all students and integrated into the whole process of personnel training. To guide the innovation and entrepreneurship education is to educate and train the innovative skills needed been produced in the colleges, and form the ecosystem among innovative knowledge, ideology and education objects. During the interactive process, the final results of "improve the quality of personnel training" show that they not only can absorb the growing nutrition provided by the education objects, but also play a more active role in the initiative and personality. They absorb nutrition, handle them and accumulate their own unique energy to gain the development of innovation and entrepreneur spirit.

Decomposers. The so-called decomposer refers to the part of decomposing and releasing the complex organic matters from the residues of animals and plants for the reusing of enterprises, which occupies an important position in the ecological system. And in the ecosystem, if the decomposers cannot lock the materials to supply the organic producers, the material recycling will be ended and the whole ecosystem will collapse. The biological role of the decomposition is not a class can be decomposed in different stages of the completion of different levels need different animals to complete. In this sense, it is not only the requirement of the diversity of the self-decomposition population, but also plays the role of "bridge" communicating the various components in the ecosystem through the "decomposition". In view of the "diversity" and "bridge", it has two important roles. The "decomposer" in the ecosystem of innovation and entrepreneurship education can be supported by education. The essence of education is to support the communication of education 
information between the producers and the consumers, and its diversity needs to give play to the educational function.

Inorganic Environment. The ecological system in the non-organic or inorganic environment, the ecology mainly refers to the light, temperature, precipitation, wind, soil and so on and influence of other factors. Environment is the basic inorganic environment of the livings. When the producer comes into the organic compound community compounded by the organisms, it will turn light energy into chemical energy. The construction and development of entrepreneurial ecosystem in colleges need a certain physical environment as the premise and foundation to provide energy. In the eco system, producers - colleges and universities will be able to produce energy to maintain the energy of ecosystem function. This is the reality of the main body of the inorganic environment, that is, there is an indispensable element among all the elements in the system, such as: social innovation and entrepreneurship atmosphere, policies and regulations of institutions, etc.

\section{To Improve and Perfect the Educational Ecosystem of Entrepreneurship and Innovation Talents in Colleges and Universities of Our Country}

First of all, we should urge the self-realization of all the relevant elements and make the elements interact positively. According to various elements, whether it is the university, the education object, or vector entrepreneurship education, the role of social system environment, there is a lot of space to exert their own potential for ecosystems. Secondly, we should promote the various relevant elements within China's entrepreneurship education system and make them maintain the trend of innovative development. Although our pioneering education is still in the exploratory stage, its importance has been widely concerned. Third, we should strengthen the coordination and communication between the external powers of the ecological system, and make them work together. The existing of ecological system must be three-dimensional and dynamic balanced. Alone or within the core of the interior of the university education system, it cannot unilaterally force rapid harmony. It must base on the good communication between social and political rights to promote the long-term stability of the ecological system.

\section{Conclusion}

Above all, it becomes more realistic, practical and feasible developmental path by taking advantages of the systematic concept of ecology and correlations among each element to facilitating the colleges and universities in our country to further explore and build innovative educational ideas and practical system with China's unique characteristics. We should make clear the responsibilities of all kinds of elements in the process of cultivating innovative talents to guide the thorough and effective development of innovative education. It is the main trend of cultivating innovative talents to construct the educational systematic ecology of innovative talents in the future, so we should pay more attention to absorbing the good practice of cultivating innovative talents in Europe and the United States. It is necessary to pay more attention to forming the systematic structure and exploration which are in accordance with China's national conditions and can meet the needs of socialist market economy, and practice earnestly and reform and create the theory according to the innovation and entrepreneurship education in our country's colleges and universities.

\section{References}

[1] Q. Zhang, L. Wu: Research on National Higher Education, 2015.

[2] D.H. Cao: Zhejiang Industry and Trade Vocational College Journal, 2015.

[3] G.H. Huang: Innovation and Entrepreneurship Education, 2015.

[4] T. Wang, J.D. Liu: Liaoning Educational Administration College Journal.2015.

[5] C.H. Wang: Continuing Education Research, 2012. 
[6] Y. Li, S.X. Liu: Research on Higher Education of Finance and Economics, 2013.

[7] H. Xue: Jiangsu Higher Education, 2014.

[8] Z. He: Renmin University of China press, 2011.

[9] Y.B. Zou:Science Press, 2011. 